\title{
Evaluation of Legionellosis Surveillance in Michigan Focusing on Diagnostic Testing
}

\author{
Leigh M. Tyndall Snow ${ }^{\star 1,2}$, Veronica A. Fialkowski ${ }^{1,2}$ and Mary Grace Stobierski ${ }^{1}$ \\ ${ }^{1}$ Michigan Department of Health and Human Services, Lansing, MI, USA; ${ }^{2}$ CDC/CSTE Applied Epidemiology Fellowship, Atlanta, GA, \\ USA
}

\section{Objective}

To describe the strengths and weaknesses of Michigan's legionellosis surveillance system and the influence of diagnostic methods on the temporal and geographic distribution of legionellosis cases in Michigan.

\section{Introduction}

In Michigan, both presentations of legionellosis, Pontiac Fever (PF) and Legionnaires' Disease (LD), are reportable through the Michigan Disease Surveillance System (MDSS), a web-based electronic database. Legionella pneumophila serogroup 1 is responsible for 50$90 \%$ of cases. ${ }^{1,2}$ Several diagnostic tests are available with varying sensitivities and specificities. Urinary Antigen testing (UAg) is the most commonly used test but only reliably detects L. pneumophila- 1 . Culturing is the gold standard test but is limited by antibiotic interference, technical expertise, and time. ${ }^{3}$ The purpose of this study was to evaluate Michigan's legionellosis surveillance system and to determine if diagnostic methods influenced case distribution.

\section{Methods}

Descriptive and quantitative analyses were conducted using suspect and confirmed legionellosis cases reported to the MDSS from 20042013. Michigan's legionellosis surveillance system was evaluated according to the 2001 MMWR surveillance system evaluation guidelines focusing on data quality, timeliness, and sensitivity. ${ }^{4} \mathrm{~A}$ survey of local health departments (LHD) was used to assess data quality, acceptability, and usefulness. The median time between each step in the reporting system was calculated and compared with state reporting requirements. Data from 2013 hospitalized LD cases reported through the MDSS were compared with 2013 Michigan hospital discharge data for LD cases to estimate the system's sensitivity. Clinical laboratories in Michigan were surveyed for their diagnostic techniques and procedures.

\section{Results}

From 2004-2013, 1756 cases of legionellosis were reported. Average annual incidences of 1.44 and 0.07 per 100,000 were calculated for LD and PF, respectively. Annual legionellosis incidence between 2004 and 2013 increased from 1.23 to 2.75 per 100,000 . The median time from diagnosis to reporting was 2 days, with a median of 14 days between case entry and completion. Among all reported cases, $13.8 \%$ of key variables had unknown or missing values; however, completeness improved by more than $20 \%$ over 10 years. Hospital discharge data recorded 284 LD cases in 2013, while the MDSS recorded 246 cases. Of these, 189 were able to be matched on birthdate and zip code, for a calculated reporting sensitivity of $67.5 \%$. Overall, $87.8 \%$ of cases were confirmed via UAg, of which $30.5 \%$ were confirmed by additional testing. Cultures were performed on $10.5 \%$ of the cases.

\section{Conclusions}

Overall, the surveillance system showed moderate sensitivity and reporting times in excess of the 24-hour state requirements. However, key variable completeness increased more than $20 \%$ over 10 years. With few cases diagnosed by culture, linking cases to an environmental source, and therefore investigating outbreaks, is challenging. The high proportion of cases confirmed by UAg alone could lead to $10-50 \%$ of cases going undiagnosed. Further research is needed to develop standardized molecular diagnostic testing methods, e.g. PCR, that are rapid, noninvasive, and comprehensive to allow for linking cases with environmental sources.

\section{Keywords}

Surveillance evaluation; Waterborne disease; Legionellosis

\section{Acknowledgments}

MDHHS Bureau of Disease Control, Prevention, and Epidemiology Staff: Jevon McFadden, MD, MPH; Shannon Johnson, MPH, Edward Hartwick, MS, Tiffany Henderson, MPH, Joyce Lai, MPH, Keira Wickliffe Berger, RN, MSN, MPH, Timothy Bolen.

MDHHS Bureau of Laboratories Staff: James Rudrik, PhD, William Crafts, MT

This study/report was supported in part by an appointment to the Applied Epidemiology Fellowship Program administered by the Council of State and Territorial Epidemiologists (CSTE) and funded by the Centers for Disease Control and Prevention (CDC) Cooperative Agreement Number 1U38OT000143-03.

\section{References}

1. Benin AL, Benson RF, Besser RE. Trends in Legionnaires' Disease, 1980-1998: Declining mortality and new patterns of diagnosis. Clin Infect Dis. 2002; 35: 1039-46.

2. Fields BS, Benson RF, Besser RE. Legionella and Legionnaires Disease: 25 years of investigation. Clin Microbiol Rev. 2002; 15(3): 506-526.

3. Murdoch DR. Diagnosis of Legionella infection. Clin Infect Dis. 2003; 36: 64-69.

4. German RR, Lee LM, Horan JM, et al. Updated guidelines for evaluating public health surveillance systems: recommendations from the Guidelines Working Group. MMWR Recomm Rep. 2001; 50 (RR-13):1-35. Available from: http://www.cdc.gov/mmwr/preview/ mmwrhtml/rr5013a1.htm.

*Leigh M. Tyndall Snow

E-mail: TyndallSnowL@michigan.gov 